\title{
Visualisation of BioPAX Networks using BioLayout Express ${ }^{3 \mathrm{D}}$ [version 1; peer review: 2 approved]
}

\author{
Derek W. Wright ${ }^{1}$, Tim Angus ${ }^{1}$, Anton J. Enright ${ }^{2}$, Tom C. Freeman ${ }^{1}$
}

${ }^{1}$ The Roslin Institute and Royal (Dick) School of Veterinary Studies, The University of Edinburgh, Midlothian, Scotland, EH25 9RG, UK

2EMBL-European Bioinformatics Institute, Wellcome Trust Genome Campus, Hinxton, Cambridge, CB10 1SD, UK

V1 First published: 20 Oct 2014, 3:246

https://doi.org/10.12688/f1000research.5499.1

Latest published: 20 Oct 2014, 3:246

https://doi.org/10.12688/f1000research.5499.1

\section{Abstract}

BioLayout Express $3 \mathrm{D}$ is a network analysis tool designed for the visualisation and analysis of graphs derived from biological data. It has proved to be powerful in the analysis of gene expression data, biological pathways and in a range of other applications. In version 3.2 of the tool we have introduced the ability to import, merge and display pathways and protein interaction networks available in the BioPAX Level 3 standard exchange format. A graphical interface allows users to search for pathways or interaction data stored in the Pathway Commons database. Queries using either gene/protein or pathway names are made via the cPath2 client and users can also define the source and/or species of information that they wish to examine. Data matching a query are listed and individual records may be viewed in isolation or merged using an 'Advanced' query tab. A visualisation scheme has been defined by mapping BioPAX entity types to a range of glyphs. Graphs of these data can be viewed and explored within BioLayout as 2D or 3D graph layouts, where they can be edited and/or exported for visualisation and editing within other tools.

Keywords

signalling network, analysis, proteins, bioinformatics

EMBL-EBI 䌈綡

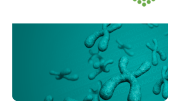

This article is included in the EMBL-EBI

collection.

\section{Open Peer Review \\ Approval Status \\ 1 \\ 2 \\ version 1 \\ 20 Oct 2014

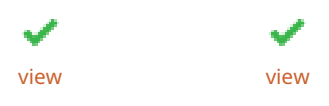 \\ 1. Gary D. Bader ID, University of Toronto, \\ Toronto, Canada \\ 2. Jianguo Xia, McGill University, Montreal, Canada \\ Any reports and responses or comments on the article can be found at the end of the article.}


Corresponding author: Derek W. Wright (Derek.Wright@roslin.ed.ac.uk)

Competing interests: No competing interests were disclosed.

Grant information: T.C.F. is funded by an Institute Strategic Grant from the Biotechnology and Biological Sciences Research Council (BBSRC) [grant number BB/JO1446X/1] and development of BioLayout Express3D is funded by BBSRC grant BB/I001107/1.

The funders had no role in study design, data collection and analysis, decision to publish, or preparation of the manuscript.

Copyright: $\odot 2014$ Wright DW et al. This is an open access article distributed under the terms of the Creative Commons Attribution License, which permits unrestricted use, distribution, and reproduction in any medium, provided the original work is properly cited. Data associated with the article are available under the terms of the Creative Commons Zero "No rights reserved" data waiver (CC0 1.0 Public domain dedication).

How to cite this article: Wright DW, Angus T, Enright AJ and Freeman TC. Visualisation of BioPAX Networks using BioLayout Express

3D [version 1; peer review: 2 approved] F1000Research 2014, 3:246 https://doi.org/10.12688/f1000research.5499.1

First published: 20 Oct 2014, 3:246 https://doi.org/10.12688/f1000research.5499.1 


\section{Introduction}

There has been an explosion in the amount of publicly available pathway and interaction data in recent years, derived from highthroughput experimental techniques, such as two-hybrid systems, mass spectrometry, phage display etc., or through focused studies and manually curated from the literature into pathway models ${ }^{1-3}$. There are many resources that store such data: at the time of writing, the website pathguide. org $^{4}$ listed 547 pathway and interaction databases. However, many of these resources store the data in idiosyncratic formats and as a result it has been difficult for resources to exchange data between them.

To address this problem, there have been a number of efforts to standardize the exchange of pathway and protein interaction data from disparate sources, including PSICQUIC ${ }^{5}$, CellML ${ }^{6}$ and BioPAX ${ }^{7}$. Of these, BioPAX is one of the most widely adopted data exchange formats. BioPAX is a community standard ontology for describing pathway and protein interaction data, suitable for qualitatively representing the current knowledge of biological systems. Seventy-four of the resources listed by the PathGuide currently support BioPAX, including some of the most widely used resources. BioPAX is overseen by the Computational Modeling in Biology Network (COMBINE) (http:// co.mbine.org/) and has been released in major versions referred to as levels. The latest release is BioPAX level 3, version 1.0.

Pathway Commons ${ }^{8}$ is a publicly available resource that aggregates and integrates pathway data from multiple organisms and databases into a common BioPAX language linked data representation. Data stored within this resource are currently derived from the databases $\mathrm{ChEBI}^{9}$, UniProt ${ }^{10}$, Reactome ${ }^{1}$, Pathway Interaction Database $^{11}$, PhosphoSite ${ }^{10}$, HumanCyc $^{12}$, HPRD $^{13}$ and PANTHER ${ }^{2}$. The Pathway Commons website provides query and bulk download of these data. The system also makes these data available via a REST web service API, which provides programmatic access to data over the web. Pathway Commons has recently been upgraded, supporting BioPAX Level 3 and providing advanced graph queries via the CPath2 REST API.

A range of software tools already supports BioPAX use and exchange. For example the network analysis tool Cytoscape ${ }^{14}$ has support via plugins (known as "apps" in Cytoscape version 3) ) $^{15,16}$. The BiNoM plugin ${ }^{17}$ can import BioPAX Level 3 OWL files, the CyPath2 plugin is able to import and visualise BioPAX from the Pathway Commons resource, and the ChiBE pathway editor ${ }^{18}$ allows users to visually edit BioPAX pathways. CellDesigner, a graphical pathway editor, can export pathways as BioPAX ${ }^{19}$ and users of the R statistical programming language can access BioPAX via the rBiopaxParser package ${ }^{20}$. As BioPAX is a language based on the semantic RDF/OWL standard, it can also be edited using standard ontology authoring tools such as Protégé and WebProtégée ${ }^{21}$. However, it should be noted that there are various compatibility issues with some of the above, with different apps/tools being specific for different versions of the tools or BioPAX.

There is considerable interest in BioPAX data from the bioinformatics community and a growing interest in tools that support its visualisation and analysis. Here we report the implementation of a simple-to-use graphical interface within the network analysis tool
BioLayout Express ${ }^{3 \mathrm{D} 22,23}$ that now supports querying of the Pathway Commons resource, allowing the user to pull in the results of specific gene/protein- or pathway-centric queries, and to visualise the results in a graphically intuitive manner.

\section{Implementation}

BioLayout Express $^{3 \mathrm{D}}$ version 3.2 has been developed to open BioPAX Level 3 OWL files and generate network graph visualisations of BioPAX encoded pathway or protein interaction data. A web service client has also been developed within BioLayout to query Pathway Commons and import BioPAX networks directly.

PaxTools $^{24}$, an open-source Java library for developing BioPAX applications, has been incorporated into BioLayout. When a BioPAX OWL file is opened, it is parsed using PaxTools and an in-memory object model containing the elements in the BioPAX document is created. If the BioPAX major version is lower than Level 3, the object model is upgraded to Level 3 before the graph is constructed. The program iterates through each entity, looking up and assigning a shape for that entity type then creating a corresponding graph node. The program then connects nodes by creation of edges to represent components that are members of a complex, steps of a pathway and participants of an interaction.

\section{Input options}

BioLayout Express ${ }^{3 \mathrm{D}}$ communicates with Pathway Commons via the cPath2 REST API, sending commands using the PaxTools library. Using a search dialog (Figure 1A), opened by selecting "File -> Network From Public Database..." within BioLayout, the user may refine searches by keywords, species, data source and BioPAX type. For convenience, predefined search options are provided for individual data sources and popular species. Queries may be for specific genes/proteins or for pathways, searching the entire record or just the title.

Search results are displayed in a table. Clicking on a search hit displays an excerpt from the description with the highlighted search term, the persistent Uniform Resource Identifier (URI) of the search hit on Pathway Commons, the number of interactions (for pathways only) and the species name, which are displayed in a panel alongside the table of hits. As the BioPAX document defines species using Identifiers.org ${ }^{25}$ standard URIs, the scientific names of the species in the search results are looked up in the NCBI Taxonomy database ${ }^{26}$ using the NCBI EFetch SOAP $^{27}$ web service. This live lookup ensures that BioLayout is capable of displaying the name of any species that may be found in the search results. For a pathway search hit, the number of interactions is calculated and displayed, so as to give the user an indication of the size of the network that will be produced (some hits may be very small and possibly not worth displaying). This value is obtained by counting the results of a traverse query of interactions within the selected pathway.

The user may choose to display the network for a single search hit, in which case BioLayout downloads the corresponding OWL file from Pathway Commons and opens it, displaying the network. Alternatively, a user may choose to select a number of search hits and then perform advanced graph queries on multiple hits, using 


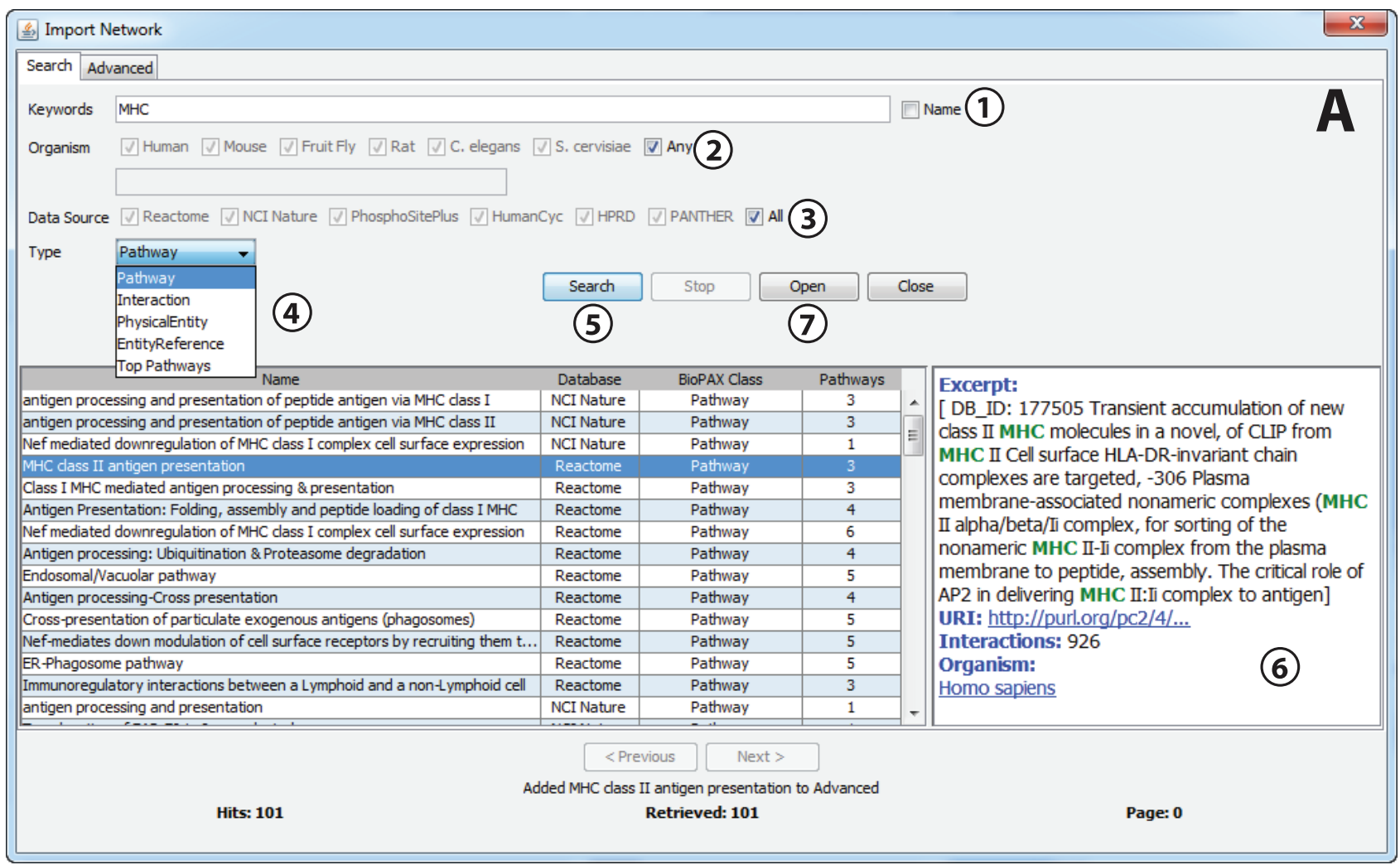

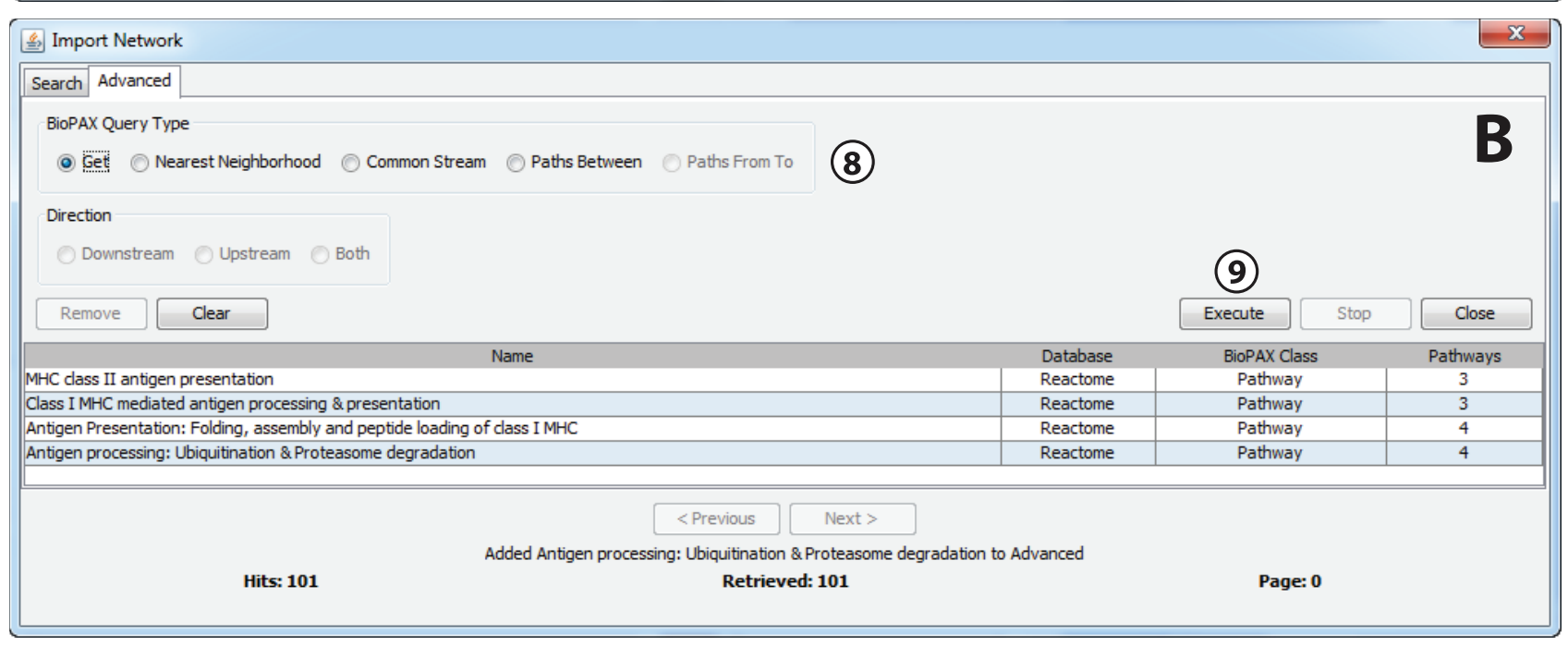

Figure 1. Import Network user interfaces with main features highlighted. (A) Import Network search dialog. This dialog supports the querying of the Pathway Commons database. 1. Keywords are entered into the Keywords field. To restrict the search to the BioPAX pathway name only (as opposed to all text associated with pathway), leave the 'Name' checkbox ticked. Terms may be separated with Boolean operators AND/OR and a specific search field may be combined with a search term separated by a colon (:) and wildcards may be searched using an asterisk $\left({ }^{*}\right) ; \mathbf{2}$. NCBI organism ID number or species name may be entered in the text field or for convenience popular species may be searched for using the checkboxes provided; $\mathbf{3}$. Information from specific databases from which Pathway Commons aggregates may be selected, restricting searches to information provided by those resources; 4. Dropdown list defines which BioPAX type to search for: Pathway, Interaction, Physical Entity, Entity Reference. There is an additional option, Top Pathways, which is a special case; this is defined as "pathways that are neither 'controlled' nor 'pathwayComponent' of another process"; 5 . Click the Search button to perform the search. Search results are displayed in the table. Results are returned in pages of 500 search hits; the sequence of pages may be navigated using the Next/ Previous buttons; 6 . Click on a row in the results table to display detailed information about that network in the pane on the right hand side; 7. Click the Open button to download and display the network for the search hit you have highlighted in the results table; 8. If you wish to perform an advanced graph query, double-click the row(s) in the results table and the search hits will be added to the Advanced tab. The Advanced tab of the Import Network dialog enables you to perform advanced graph queries on search merging networks etc.; 9. When the procedure is defined, click Execute to visualise the results. 
the operations provided by the Pathway Commons cPath2 web service. A search hit may be added to the Advanced tab by doubleclicking the row (Figure 1B). Advanced query options are:

- Get-multiple networks combined into a single network

- Nearest Neighborhood - first order neighborhood of nodes within search hits

- Common Stream - common upstream or downstream of search hits

- Paths Between - network forming paths between search hits

- Paths From To - network forming the paths from search hits in rows selected by the user in the Advanced table to unselected search hits

\section{Visualisation of BioPAX data}

A visual scheme has been defined, where node shapes and colours have been mapped to BioPAX entity types. BioLayout already supports the import of mEPN (modified Edinburgh Pathway Notation) pathway models ${ }^{3,28}$ saved as GraphML files. When visualised, the concepts supported by this pathway notation system are translated into equivalent $2 \mathrm{D}$ or $3 \mathrm{D}$ shapes. We therefore chose equivalent glyphs for BioPAX entities and concepts where possible, in order to provide a consistent user experience.

Some BioPAX concepts could not be mapped to the existing mEPN scheme so new glyphs were added. For example, a dumbbell shape was added for RNA-Region. Some concepts did not have an exact analogue. In the case of the BioPAX Small Molecule type, the equivalent concept in mEPN could either be Ion/Simple Molecule or Simple Biochemical; the Ion/Simple Molecule glyph was used. The BioPAX ontology has a hierarchical structure with increasing levels of granularity. Some glyphs were added to mEPN to handle generic BioPAX types where the more detailed type is not available in the data, such as a Control transition. Mappings between BioPAX/mEPN concepts and the $2 \mathrm{D}$ and $3 \mathrm{D}$ shapes used to represent them are shown in Figure 2.

\section{Layout}

BioPAX may describe the interaction between the components of a pathway but it does not define layout co-ordinates for visualisations, even if the original source of the information, such as Reactome, contained this information. In the absence of layout information, a graph layout must be computed algorithmically. We recommend the Fast Multipole Multilevel Method (FMMM) layout algorithm, implemented within BioLayout, for use with BioPAX networks ${ }^{29}$. FMMM is a force-directed layout algorithm, introduced in BioLayout version 3.1 that allows graph layout to be computed highly efficiently, with a small number of iterations. The algorithm produces elegantly laid out graphs (Figure 3A) in both 2D and 3D, with sparsely arranged nodes and is particularly useful for the visualisation of large structured networks, such as those obtained from BioPAX.

\section{Exploration of networks}

BioLayout's Class Viewer ${ }^{23}$ is used as an inspector for the graph. The Class Viewer enables a graph to be sub-categorized into classes, based on node type. The classes taken together form a Class Set. During the graph construction process, a Class Set is created for BioPAX features and as the graph nodes are created, each node is assigned to a class with the name of the BioPAX entity type to which it corresponds (Figure 3B).

The Import Network search dialog may be opened from within the Class Viewer, while navigating a gene co-expression network, using the Search Database function. This opens the dialog with the Keywords field pre-populated with gene names from selected

\begin{tabular}{|l|l|l|}
\hline BioPAX & MEPN \\
\hline Physical Entity & Generic Entity & \\
\hline Complex & Protein Complex & \\
\hline Dna & DNA & \\
\hline DnaRegion & DNA Region & \\
\hline Protein & Protein Peptide & \\
\hline Rna & RNA & \\
\hline RnaRegion & RNA Region & \\
\hline SmallMolecule & Ion Simple Molecule & \\
\hline Gene & Gene & \\
\hline Pathway & Pathway Module \\
\hline
\end{tabular}

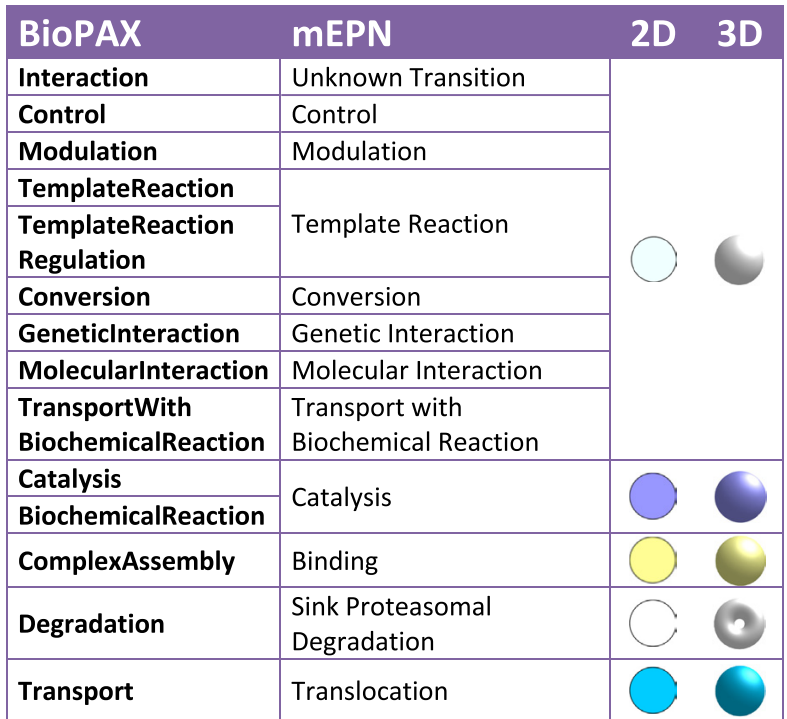

Figure 2. Table listing BioPAX concepts and the mapping on to the mEPN pathway notation scheme and their representation in BioLayout Express ${ }^{3 \mathrm{D}}$ as 2D and 3D nodes. 


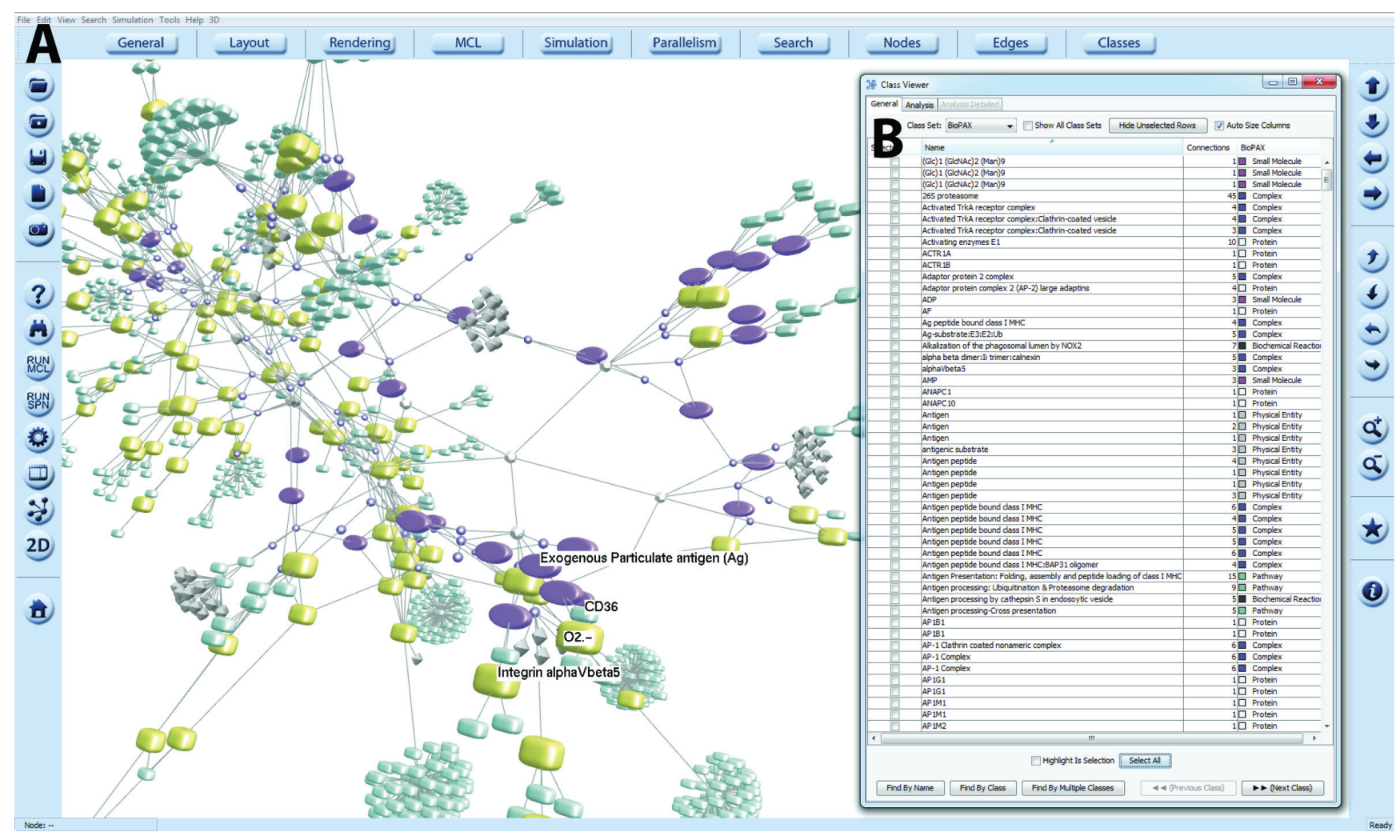

Figure 3. Visualisation of BioPAX networks in BioLayout Express ${ }^{3 \mathrm{D}}$. (A) 3D network view of a BioPAX query. Different entity/concept types defined in BioPAX Level 3 are translated into nodes of different size, shape and colour depending on type. Graphs may be explored, edited and names displayed. Options for these functionalities are contained within the BioLayout menus. (B) The names and node types of selected nodes may be viewed with the Class Viewer, which also provides information on the number of edges. Within this window nodes may be selected/deselected, searched by name or class and information exported.

nodes within the graph, enabling the user to search for pathways that involve the genes of interest. This represents a means of directly integrating genomic data with pathway data. Similarly, an analysis of a gene expression network or similar, e.g. clustering of co-expression modules, may be exported (File $->$ Export $->$ Class Sets As File) and then, assuming that the node identifiers are the same between the two networks, imported (File -> Import -> Class Sets...) whilst visualising a BioPAX network. In this way, the genes of interest in the expression network can be highlighted on the interaction network or vice versa.

\section{Conclusion}

There is now a wealth of pathway and protein interaction data in the public domain, collected and curated at great expense. However, accessing and using these data has proved challenging for many due to the lack of standard formats for data exchange between resources. The BioPAX standard has gone a long way to resolve this issue and has been widely adopted by the community. The Pathway Commons database has therefore been able to amalgamate the information stored in a number of the main pathway/ interaction resources, making the information available through the CPath2 web service. Here we report our implementation of data query and import functionality within BioLayout Express ${ }^{3 \mathrm{D}}$ version 3.2, thereby leveraging a powerful tool to support the visualisation and analysis of large pathway and protein interaction networks. The data stored in the Pathway Commons resource may now be easily searched and hits combined. The resulting networks can be displayed in $2 \mathrm{D}$ or $3 \mathrm{D}$ using a graphical display language that differentiates between the entity types described in the BioPAX hierarchy. Within the tool, the graphs can be explored and edited and where necessary exported for visualisation within other tools.

\section{Software availability \\ Sofware access}

Software and source code are available from http://www.biolayout.org.

Archived source code as at the time of publication http://dx.doi.org/10.5281/zenodo. $12216^{30}$ 


\section{Software license}

GNU Public Licence version 3 http://www.gnu.org/copyleft/gpl. html

\section{Author contributions}

D.W.W. and T.A. implemented the software described in this paper and A.J.E. advised and assisted with the implementation. T.C.F. oversaw the work described here and co-authored the paper with D.W.W.

\section{Competing interests}

No competing interests were disclosed.

\section{Grant information}

T.C.F. is funded by an Institute Strategic Grant from the Biotechnology and Biological Sciences Research Council (BBSRC) [grant number BB/JO1446X/1] and development of BioLayout Express ${ }^{3 \mathrm{D}}$ is funded by BBSRC grant BB/I001107/1.

The funders had no role in study design, data collection and analysis, decision to publish, or preparation of the manuscript.

\section{Acknowledgements}

We would like to thank Igor Rodchenkov, Emek Demir and Gary Bader for valuable support with implementing Pathway Commons and PaxTools within BioLayout Express ${ }^{3 \mathrm{D}}$.
1. Croft D, Mundo AF, Haw R, et al.: The Reactome pathway knowledgebase. Nucleic Acids Res. 2014; 42(Database issue): D472-7. PubMed Abstract | Publisher Full Text | Free Full Text

2. Mi H, Muruganujan A, Thomas PD: PANTHER in 2013: modeling the evolution of gene function, and other gene attributes, in the context of phylogenetic trees. Nucleic Acids Res. 2013; 41(Database issue): D377-D86. PubMed Abstract | Publisher Full Text | Free Full Text

3. Raza S, McDerment N, Lacaze PA, et al:: Construction of a large scale integrated map of macrophage pathogen recognition and effector systems. BMC Syst Biol. 2010; 4: 63

PubMed Abstract | Publisher Full Text | Free Full Text

4. Bader GD, Cary MP, Sander C: Pathguide: a pathway resource list. Nucleic Acids Res. 2006; 34(Database issue): D504-D6. PubMed Abstract | Publisher Full Text | Free Full Text

5. Aranda B, Blankenburg H, Kerrien S, et al:: PSICQUIC and PSISCORE: accessing and scoring molecular interactions. Nat Methods. 2011; 8(7): 528-9. PubMed Abstract | Publisher Full Text | Free Full Text

6. Lloyd CM, Halstead MD, Nielsen PF: CellML: its future, present and past. Prog Biophys Mol Biol. 2004; 85(2-3): 433-50. PubMed Abstract | Publisher Full Text

7. Demir E, Cary MP, Paley S, et al:: The BioPAX community standard for pathway data sharing. Nat Biotechnol. 2010; 28: 935-42. PubMed Abstract | Publisher Full Text | Free Full Text

8. Cerami EG, Gross BE, Demir E, et al.: Pathway Commons, a web resource for biological pathway data. Nucleic Acids Res. 2011; 39(Database issue): D685-90. PubMed Abstract | Publisher Full Text | Free Full Text

9. Degtyarenko $\mathrm{K}$, de Matos $\mathrm{P}$, Ennis M, et al:: ChEBI: a database and ontology for chemical entities of biological interest. Nucleic Acids Res. 2008; 36(Database issue): D344-50.

PubMed Abstract | Publisher Full Text | Free Full Text

10. Hornbeck PV, Chabra I, Kornhauser JM, et al.: PhosphoSite: A bioinformatics resource dedicated to physiological protein phosphorylation. Proteomics. 2004; 4(6): 1551-61

PubMed Abstract | Publisher Full Text

11. Schaefer CF, Anthony K, Krupa S, et al: PID: the Pathway Interaction Database. Nucleic Acids Res. 2009; 37(Database issue): D674-9. PubMed Abstract | Publisher Full Text | Free Full Text

12. Romero P, Wagg J, Green ML, et al:: Computational prediction of human metabolic pathways from the complete human genome. Genome Biol. 2005; 6(1): R2. PubMed Abstract | Publisher Full Text | Free Full Text

13. Prasad TS, Kandasamy K, Pandey A: Human Protein Reference Database and Human Proteinpedia as discovery tools for systems biology. Methods $\mathrm{Mol}$ Biol. 2009; 577: 67-79.

PubMed Abstract | Publisher Full Text

14. Shannon P, Markiel A, Ozier O, et al:: Cytoscape: a software environment for integrated models of biomolecular interaction networks. Genome Res. 2003 ; 13(11): 2498-504.

PubMed Abstract | Publisher Full Text | Free Full Text

15. Lotia S, Montojo J, Dong Y, et al.: Cytoscape app store. Bioinformatics. 2013 29(10): 1350-1.

PubMed Abstract | Publisher Full Text | Free Full Text
16. Saito R, Smoot ME, Ono K, et al.: A travel guide to Cytoscape plugins. Nat Methods. 2012; 9(11): 1069-76.

PubMed Abstract | Publisher Full Text | Free Full Text

17. Bonnet E, Calzone L, Rovera D, et al.: BiNoM 2.0, a Cytoscape plugin for accessing and analyzing pathways using standard systems biology formats. BMC Syst Biol. 2013; 7: 18.

PubMed Abstract | Publisher Full Text | Free Full Text

18. Babur $\mathrm{O}$, Dogrusoz $U$, Cakır M, et al.: Integrating biological pathways and genomic profiles with ChiBE 2. BMC Genomics. 2014; 15: 642.

PubMed Abstract | Publisher Full Text | Free Full Text

19. Mi H, Muruganujan A, Demir E, et al.: BioPAX support in CellDesigner. Bioinformatics. 2011; 27(24): 3437-8.

PubMed Abstract | Publisher Full Text | Free Full Text

20. Kramer F, Bayerlová M, Klemm F, et al.: RBiopaxParser--an R package to parse, modify and visualize BioPAX data. Bioinformatics. 2013; 29(4): 520-2. PubMed Abstract | Publisher Full Text

21. Horridge $\mathrm{M}$, Tudorache $\mathrm{T}$, Nuylas $\mathrm{C}$, et al:: WebProtege: a collaborative Web Based platform for editing biomedical ontologies. Bioinformatics. 2014; 30(16) 2384-5.

PubMed Abstract | Publisher Full Text | Free Full Text

22. Freeman TC, Goldovsky L, Brosch M, et al.: Construction, visualisation, and clustering of transcription networks from microarray expression data. PLOS Comput Biol. 2007; 3(10): 2032-42.

PubMed Abstract | Publisher Full Text | Free Full Text

23. Theocharidis A, van Dongen S, Enright AJ, et al:: Network visualization and analysis of gene expression data using BioLayout Express(3D). Nat Protoc. 2009; 4(10): 1535-50.

PubMed Abstract | Publisher Full Text

24. Demir E, Babur Ö, Rodchenkov I, et al:: Using Biological Pathway Data with Paxtools. PLOS Comput Biol. 2013; 9(9): e1003194. PubMed Abstract | Publisher Full Text | Free Full Text

25. Juty N, Le Novere N, Laibe C: Identifiers.org and MIRIAM Registry: community resources to provide persistent identification. Nucleic Acids Res. 2012; 40(Database issue): D580-6.

PubMed Abstract | Publisher Full Text | Free Full Text

26. Federhen S: The NCBI Taxonomy database. Nucleic Acids Res. 2012; 40(Database issue): D136-43.

PubMed Abstract | Publisher Full Text | Free Full Text

27. NCBI Resource Coordinators: Database resources of the National Center for Biotechnology Information. Nucleic Acids Res. 2014; 42(Database issue): D7-17. PubMed Abstract | Publisher Full Text | Free Full Text

28. Freeman TC, Raza S, Theocharidis A, et al.: The mEPN scheme: an intuitive and flexible graphical system for rendering biological pathways. BMC Syst Biol. 2010; 4: 65

PubMed Abstract | Publisher Full Text | Free Full Text

29. Angus T, Freeman TC, Klein K editors. Application of Graph Layout Algorithms for the Visualization of Biological Networks in 3D. Graph Drawing: Springer. Reference Source

30. Wright DW, Angus T, Enright AJ, et al.: BioLayout Express ${ }^{3 \mathrm{D}}$ Version 3.2. Zenodo 2014. Data Source 


\section{Open Peer Review}

\section{Current Peer Review Status:}

\section{Version 1}

Reviewer Report 07 April 2015

https://doi.org/10.5256/f1000research.5869.r8200

(c) $2015 \mathrm{Xia}$ J. This is an open access peer review report distributed under the terms of the Creative Commons Attribution License, which permits unrestricted use, distribution, and reproduction in any medium, provided the original work is properly cited.

\section{Jianguo Xia}

Institute of Parasitology and Department of Animal Science, Faculty of Parasitology, McGill University, Montreal, QC, Canada

General Comments:

The BioLayout Express 3D is a very useful tool, especially its unique 3D rendering capability. The supports for importing network files in BioPAX level 3 format and integration with Pathway Commons appear to work well as described in the paper. My comments are mainly concerned with network visualization.

1. Navigation in large/dense networks:

Users often want to zoom in to a particular node/region of the network to view the connections and structures. It is difficult to achieve this using right-mouse button. A better approach may be to add support for three-wheel mouse - point the mouse to the node and then scroll to zoom in to the pointer direction.

2. Enhancing support for node search in a dense network:

Users should be able to click to highlight AND zoom in to it.

3. Better scaling control (esp. for 2D view):

Users would expect that node overlapping issue would be resolved by zooming in to the region. This is not the case during testing. Rather than simply getting bigger in size, both the sizes and relatives distance between nodes should be scaled properly when users zoom in. If there are some advanced options to achieve this effect, the default parameters should be improved.

Bugs (Version 3.3, Mac version)

On the 2D view, select and drag a node to a new position, the mouse pointer and the node are not well synchronized during the process. Black screen also occurs sometimes. 
Additional comments / wish list:

1. In addition to comparing their abilities in supporting BioPAX format, a general discussion on network visualization features of these popular tools will be very useful for new users to make informed choices.

2. The standalone tools have the advantage for dealing with large data. The current tool can be improved with regard to more layout options and better performance for large networks.

3. Given the increasing popularity for web-based visualization, the support for exporting the graph for D3.js rendering is certainly a welcome step. An online version for 3D network visualization (maybe based on WebGL?) will be highly received by the community.

Competing Interests: No competing interests were disclosed.

\section{I confirm that I have read this submission and believe that I have an appropriate level of expertise to confirm that it is of an acceptable scientific standard.}

Reviewer Report 27 October 2014

https://doi.org/10.5256/f1000research.5869.r6455

(C) 2014 Bader G. This is an open access peer review report distributed under the terms of the Creative Commons Attribution License, which permits unrestricted use, distribution, and reproduction in any medium, provided the original work is properly cited.

\section{Gary D. Bader}

Donnelly Centre for Cellular and Biomolecular Research (CCBR), University of Toronto, Toronto, ON, M5S 3E1, Canada

The authors describe BioPAX loading and visualization support for the established BioLayout Express 3D network visualization software. As far as I know, this is the only software capable of 3D display of BioPAX derived networks. The new feature seems to work very well as described. I have a few minor comments to improve the manuscript.

Minor notes:

"the persistent Uniform Resource Identifier (URI)" - URIs in Pathway Commons are not guaranteed to be consistent between releases. They can be persistent if they are externally standardized e.g. uniprot IDs, but internal pathway commons URIs may change from release to release.

The manuscript would benefit from one biological use case. The highlighting expression data paragraph is interesting, but including a biological example would help potential biologist users know how to use the software for a particular analysis.

It would be nice to see a future directions section e.g. is there a plan to visualize SIF 
networks derived from BioPAX?

It would be useful to briefly mention some examples of information that is not covered in the BioPAX to mEPN mapping e.g. post-translational modifications on proteins, the directionality of edges e.g. that could distinguish between left and right participants of an interaction vs. all participants of an interaction. Some of this information is possible to show in other visualization options, such as the Systems Biology Graphical Notation, which the authors may want to reference.

Software comments:

The top pathways feature requires entering a keyword before the Search button is active, but with this query, it seems that a keyword is not necessary, as any query always shows all top pathways.

A number of nodes have 'null' labels e.g. if they are interaction nodes. It would be nice to be able to suppress these labels somehow.

- It would be useful to see other information associated with the nodes e.g. data source, links to external databases. Some of this information is already shown in section 6 in panel A of the import network dialog shown in Figure 1.

Is it possible to choose another layout after a network has been loaded?

Bug: Clicking the 'i' information button on the right hand button tool bar brings up a help dialog called the 'navigation wizard'. The links to PDFs at the bottom of this screen go to non-existent web pages.

The edge names are all ' 0 ', but could be the type of edge.

Minor text fixes:

"within BioLayout, the user may refine searches by keywords, species, data source and BioPAX type" -> BioPAX class type.

Competing Interests: I work on the Pathway Commons project that this paper makes use of. We have provided some technical support for the implementation of this work. We were not otherwise involved in this work.

I confirm that I have read this submission and believe that I have an appropriate level of expertise to confirm that it is of an acceptable scientific standard. 
The benefits of publishing with F1000Research:

- Your article is published within days, with no editorial bias

- You can publish traditional articles, null/negative results, case reports, data notes and more

- The peer review process is transparent and collaborative

- Your article is indexed in PubMed after passing peer review

- Dedicated customer support at every stage

For pre-submission enquiries, contact research@f1000.com 